\title{
Predicting the regional onset of the rainy season in West Africa
}

\author{
Patrick Laux ${ }^{1}$, Harald Kunstmann ${ }^{1} \&$ András Bárdossy \\ ${ }_{2}^{2}$ Institute for Hydraulic Engineering, University of Stuttgart, Germany
}

\section{Motivation}

Particularly in regions, where precipitation is limited to a few months per year, the reliable determination of the onset of the rainy season and the start of the sowing time is of crucial importance for sustainable food production. For farmers in the Volta Basin, the onset of the rainy season (ORS) is the most important variable: Planting too early is enhancing the risk of total crop failure, and planting too late is reducing valuable vegetation time (Laux et al., 2008). Due to a very high spatial and temporal variability of precipitation amounts, a non-uniform distribution of the rains during the rainy season and a high variability in the onset dates, farmers have problems to decide when to start with the sowing preparations. In the Volta Basin, the onset seldom occurs abruptly and is often preceded by short isolated showers with intermittent dry spells of various lengths, which are often misinterpreted as the start of the rains (false starts).

\section{Methodologies}

- Development and application of two different ORS definitions, for past mode (1961-1999) and predictive mode (current season)

- Regionalization via s-mode Principle Component Analysis (PCA) due to high spatial rainfall variability

- Linear Discriminant Analysis to judge day by day whether the ORS has already begun

- Linear Regression Analysis to identify the temporal shift of the ORS dates between the regions

- Multi Objective Fuzzy Rule-Based Classification (MOFRBC) of large-scale meteorological fields to identify weather patterns which are significantly linked with the ORS (not presented here, see therefore Laux et al., 2007)

\section{Results}

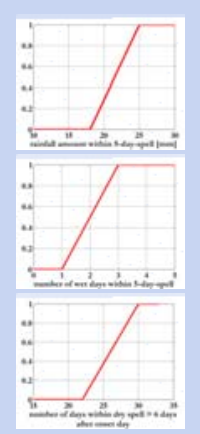

Fig. 1: Membership where:
functions of the ORS

\section{definition.}

$\Gamma=\mathrm{\gamma} 1 \cdot \mathrm{\gamma} 2 \cdot \mathrm{\gamma} 3>$ threshold (past mode)

$\Gamma=\mathrm{\gamma} 1 \cdot \mathrm{\gamma} 2>$ threshold (predictive mode)

Fig. 1 is showing the membership functions of the ORS definition accounting for the most important agricultural aspects. The ORS is calculated as the first day of year (DOY),$$
\text { restis }
$$

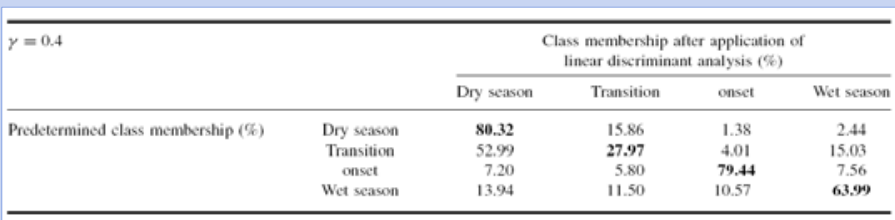

Tab. 1: Confusion matrix for the classes Dry season, Transition, Onset and Wet Season, exemplarily illustrated for
region PC4. The hit ratio [\%] (correct classifications) can be found on the Tab. 1 is exemplarily presenting the hit ratio of PC4 for the prediction of the classes dry season, transition, onset and wet season using Linear Discriminant Analysis. The hit ratios for the ORS class are ranging between $60 \%$ and $80 \%$ for the different regions.
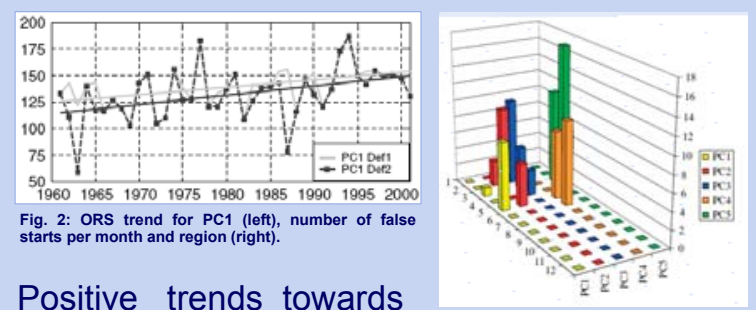

Positive trends towards

delayed regional ORS dates were observed using both definitions (Fig.2, left). PC5 is showing the highest number of false starts in April, and thus, the highest probability of total crop failure (Fig.2, right).

\section{References}

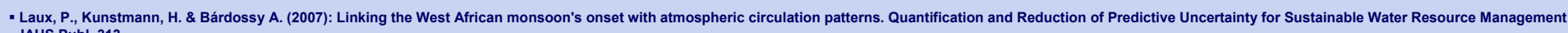
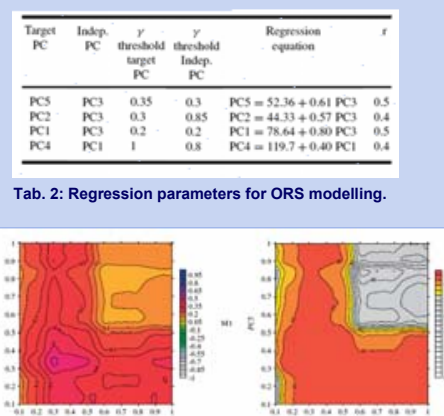

Fig. 4: Correlation coefficient of the ORS dates between PC5 and PC3 (left) using different $\Gamma$ threshold combinations (see
Fig.1) and level of significance (right). Best mutual

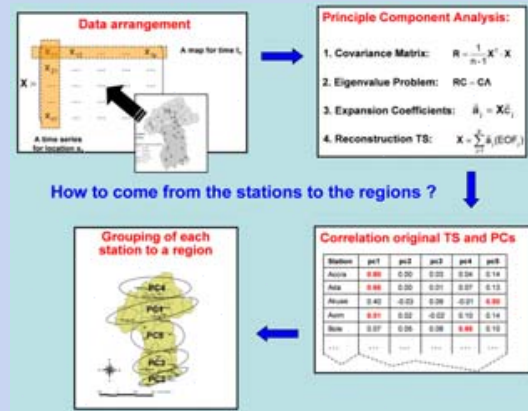

Fig. 3: Regionalization strategy applied for the Volta Basin using PCA based on the covariance matrix. Five regions with similar temporal
rainfall characteristics were obtained (see therefore Laux et al., 2008).
The grouping was obtained via correlation analysis of the original time series and the PC loadings.

LRA was applied to estimate the regional ORS dates for the ongoing season. Tab. 2 shows the estimated models. The model quality is depending strongly on the mutual $\Gamma$ combination (Fig. 5) of the definition. The correlation coefficients of the regional ORS dates were calculated to identify hot spots to improve the models. 